EXTENDED REPORT

\title{
Optic disc topographic parameters measured in the normal cynomolgus monkey by confocal scanning laser tomography
}

\author{
T Taniguchi, M Shimazawa, M Araie, G Tomita, M Sasaoka, Y Kitazawa, H Hara
}

Br J Ophthalmol 2005;89:1058-1062. doi: 10.1136/bjo.2004.062513

See end of article for authors' affiliations

....................

Correspondence to: Hideaki Hara, PhD, Department of Biofunctional Molecules, Gifu Pharmaceutical University, 5-6-1

Mitahora-higashi, Gifu 502-8585, Japan; hidehara@gifu-pu.ac.jp

Accepted for publication 1 February 2005

\begin{abstract}
Aim: To study optic disc topographic parameters in normal cynomolgus monkeys by Heidelberg retina tomograph (HRT).

Methods: 12 optic disc topographic parameters were investigated in 36 normal eyes in 18 male monkeys. Mean (SD) and interocular differences were obtained for each parameter from three independent measurements made during a 1 week period. Correlations among the topographic parameters were analysed, too.

Results: No significant differences between right and left eyes were detected for any topographic parameters. Disc area, rim area, and height variation contour showed smaller right-left differences than other parameters. The coefficients of variation for rim area, height variation contour, rim volume, mean cup depth, maximum cup depth, mean retinal nerve fibre layer (RNFL) thickness, and RNFL cross section area were less than $10 \%$ (for rim area, less than 5\%). Rim area and height variation contour showed relatively weak interrelations and neither showed a correlation with disc area.

Conclusion: For evaluating time related changes in the optic disc by HRT in monkeys, rim area and height variation contour might be useful parameters because coefficients of variation and right-left differences were lower than for other parameters and because these parameters showed weak interrelations and no correlation with disc area.
\end{abstract}

n cynomolgus and rhesus monkeys, the optic nerve head $(\mathrm{ONH})$ resembles the human $\mathrm{ONH}$ in appearance and the pathophysiological changes that occur in experimental glaucoma resemble those in patients with glaucoma. ${ }^{1-4}$ Hence, the monkey eye is a useful model for the study of glaucoma. Half the 30 or so reports on $\mathrm{ONH}$ morphology in monkeys have noted ONH changes in experimental glaucoma in cynomolgus or rhesus monkeys. Many ONH studies in glaucomatous monkeys have employed in traditional methodologies, such as fundus photography and histological analysis, and have revealed enlargement of the optic disc cup, localised retinal nerve fibre defects, and selective loss of ganglion cells with thinning of the nerve fibre layer in experimental glaucoma eyes. ${ }^{1-9}$ On the other hand, Jonas and colleagues ${ }^{10}$ used fundus photographs to evaluate the normal optic disc as a prerequisite for a proper understanding of experimentally induced optic nerve changes such as those associated with glaucomatous damage in rhesus monkeys. However, these methods do not provide a full quantitative evaluation because optic disc parameters obtained from fundus photographs are usually expressed in relative terms and, moreover, histological comparisons may be affected by tissue shrinkage. ${ }^{1-10}$ In the clinical assessment of the optic disc from fundus photographs it is difficult to be objective; indeed, results vary particularly between different observers, even if they have adequate experience. ${ }^{11}$ Furthermore, two dimensional analysis from fundus photographs may be unable to detect subtle changes in the progression of glaucoma, because glaucomatous changes in the optic disc are three dimensional.

By contrast, confocal scanning laser tomography allows us to obtain three dimensional images of the human optic disc. $^{12}{ }^{13}$ This device has been used to make quantitative, objective measurements of $\mathrm{ONH}$ structures in humans with reasonable reproducibility and potential advantages over other techniques. ${ }^{12-17}$ Recently, this method has been used to study ONH structure in monkeys. ${ }^{18-22}$ The monkey ONH was studied using a computerised digital image analyser, rather than confocal scanning laser tomography. ${ }^{23-26}$ HRT (Heidelberg retina tomograph, Heidelberg Engineering), which operates on tomographic principles, is the most widely used optic nerve imaging tool in glaucoma clinics. ${ }^{12}{ }^{14-17}$ Yücel and colleagues ${ }^{21}$ reported quantitative differences in the optic disc topographic parameters measured using HRT between the control fellow and experimental glaucoma eyes in cynomolgus monkeys. However, for a proper study of $\mathrm{ONH}$ changes in cynomolgus monkeys using HRT it is necessary to know the reproducibility of the measurements and also the variation in HRT parameters between individual normal monkeys. Further, the information about interocular differences (right-left differences) in normal monkeys is essential to use the fellow eye as a control for the experimental glaucoma eye. To our knowledge, however, appropriate data have yet to be published. We examined (a) the reproducibility of HRT measurements in a total of 36 eyes in 18 normal monkeys, (b) the interindividual variability and the right-left differences in each parameter, and (c) correlations among HRT parameters.

\section{MATERIALS AND METHODS \\ Animals}

Eighteen normal male cynomolgus monkeys (Macaca fascicularis) aged between 4.0 years and 5.5 years, weighing $4.0-$

Abbreviations: CA, cup area; C/D, cup/disc area ratio; CSM, cup shape measure; CV, cup volume; DA, disc area; HRT, Heidelberg retina tomograph; HVC, height variation contour; IOP, intraocular pressure; $M n C D$, mean cup depth; MnRNFLT, mean RNFL thickness; MxCD, maximum cup depth; ONH, optic nerve head; RA, rim area; RCSA, RNFL cross section area; RNFL, retinal nerve fibre layer; RV, rim volume 
$5.0 \mathrm{~kg}$ were used. None of the eyes had been used in any other experiment, undergone any surgical procedure, or received any medical treatment. Monkeys were housed in an air conditioned room $\left(24^{\circ} \mathrm{C} \quad\left(\mathrm{SD} \quad 2^{\circ} \mathrm{C}\right), 60 \% \quad(10 \%)\right.$ humidity) and allowed food and water ad libitum. All investigations were in accordance with the ARVO statement for the use of animals in ophthalmic and vision research.

\section{Experimental protocol}

Intraocular pressure (IOP) was measured using a calibrated pneumatonometer under ketamine anaesthesia $(8.75-10 \mathrm{mg} / \mathrm{kg}$, intramuscular: im), with local anaesthesia before any optic disc topographic images were taken. Optic disc topographic parameters were analysed using HRT (Heidelberg Engineering, Heidelberg, Germany) under ketamine (8.75$10 \mathrm{mg} / \mathrm{kg}$, im) plus xylazine $(0.5 \mathrm{mg} / \mathrm{kg}, \mathrm{im})$ anaesthesia. Before topographic image acquisition, the refractive index and corneal curvature radius were measured in each eye, then entered into the patient data submenu for the examined eye to allow correction of magnification effects on the images. The SD, which indicates the precision of the measurement process, was always less than $30 \mu \mathrm{m}$. The disc contour line was determined by an experienced operator while viewing fundus photographs. The following 12 optic disc topographic parameters were investigated: disc area (DA), cup area (CA), cup/disc area ratio (C/D), rim area (RA), height variation contour (HVC), cup volume $(\mathrm{CV})$, rim volume (RV), mean cup depth (MnCD), maximum cup depth (MxCD), cup shape measure (CSM), mean retinal nerve fibre layer (RNFL) thickness (MnRNFLT), and RNFL cross section area (RCSA). The position of the reference plane was $50 \mu \mathrm{m}$ posterior to the mean retinal height between 350 degrees and 356 degrees (temporal) along the operator drawn contour line delineating the optic disc margin. Topographic parameters were obtained three times over a l week period as independent measurements. The disc contour line was defined during the first measurement and this definition was transported to the topographic images obtained during the second and three measurements. Right-left differences in topographic parameters were calculated for each animal as the absolute difference between two eyes as previously described. ${ }^{27}$ Measurements of HRT parameters and data analysis were each performed by an operator who was masked as to the purpose of this study.

\section{Statistical analysis}

Correlations analyses were assessed using Pearson's correlation test. A Student's $t$ test was used to compare topographic parameters between the right and left eyes.

\section{RESULTS}

The fundus photographs showed no obvious retinal lesions, nerve fibre layer defects, or abnormalities of either the $\mathrm{ONH}$ or vascular structures in any of the eyes examined (fig lA). Topographic images of the optic disc were obtained using HRT (figs $1 B$ and $1 C$ ). The IOP values (mean (SD) $\mathrm{n}=18$ ) obtained for the right and left eyes were 20.7 (3.8) $\mathrm{mm} \mathrm{Hg}$ and 20.0 (3.2) $\mathrm{mm} \mathrm{Hg}$, respectively. No statistically

Table 1 Refraction in normal cynomolgus monkey eyes

\begin{tabular}{llll}
\hline & \multicolumn{2}{l}{ Right eye } & Left eye \\
\cline { 2 - 2 } & Mean (SD) & Mean (SD) \\
\hline Refraction (dioptres) & $1.01(1.52)$ & $1.27(1.45)$ \\
\hline
\end{tabular}

No significant difference between right and left eyes. Each value represents mean (SD) for 18 eyes. significant correlations were observed between any topographic parameters and IOP (data not shown). The mean data of refraction in right and left eyes are shown in table 1 . There was no significant difference between right and left eyes.

The mean (SD) values and right-left differences for each topographic parameter are shown in table 2. No statistically significant differences between the two eyes were found in all topographic parameters. Right-left differences were relatively large ( $>40 \%$ of mean value for right eye) for CA, C/D, and CV but relatively small (about $10 \%$ or $<10 \%$ of mean for right eye) for DA, RA, and HVC. On the basis of data (mean (SD), and sample size) in table 2, we calculated the statistical power for detecting a $20 \%$ right-left difference in each parameter using a Student's $t$ test. Statistical power was more than $80 \%$ for RA, HVC, MnRNFLT, and RCSA. Thus, under our experimental conditions, a statistically significant difference would be detected in several parameters with a fair degree of certainty if the difference between right and left eyes was about $20 \%$.

Seven topographic parameters (RA, HVC, RV, MnCD, MxCD, MnRNFLT, and RCSA) had coefficient of variation less than $10 \%$ for both eyes, with the smallest coefficient of variation $(<5 \%)$ in each eye being shown by RA (table 3$)$. For $\mathrm{CA}, \mathrm{C} / \mathrm{D}, \mathrm{CV}$, and CSM, the coefficient of variation was more than $10 \%$ in each eye, with that for CV being the highest (30$40 \%)$.

Finally, correlations among HRT parameters were examined (on the basis of the mean of three independent measurements from each eye) using Pearson's correlation coefficient (table 4). CA, C/D, CV, RV, and MnCD displayed relatively strong interrelations (correlation coefficient more than 0.7), with significance being demonstrated for each eye, while DA, RA, HVC, and CSM exhibited relatively weak interrelations.

\section{DISCUSSION}

In this study, we measured optic disc topographic parameters in normal monkey eyes by means of HRT. The mean values obtained for each HRT parameter in the right and left eyes are comparable to those obtained for control fellow eyes in the previous study of experimental glaucoma in cynomolgus monkeys. ${ }^{21}$ Moreover, the coefficient of variation for parameters related to the optic disc cup (namely, CA, C/D, CV, and CSM) exceeded $10 \%$, which is compatible with findings reported by others for humans. ${ }^{28}$ By contrast, RA, HVC, RV, MnCD, MxCD, MnRNFLT, and RCSA each had coefficient of variation less than $10 \%$ for both eyes than those relating to the optic disc cup, suggesting that the values obtained for these parameters may be more reliable in monkeys. The coefficient of variation for RA was particularly low in this study and, interestingly, Tan and colleagues ${ }^{28}$ noted that RA was the most reproducible parameter in humans. Previous reports have indicated that RA correlates well with the visual field and the degree of glaucomatous optic disc damage in humans. ${ }^{15-17}$ These findings suggest that RA should be suited for the evaluation of glaucomatous changes using HRT in monkey eyes as well as in humans.

There are two ways to define the disc contour line for evaluating time related changes in HRT parameters. One is to transport the contour line obtained for the base HRT image to subsequent images, the other is to redefine the contour line for each image. Roff et al compared the data variability between these two methods. ${ }^{29}$ Their results indicated that the use of transport led to a lower data variability than redefinition. When investigating the coefficient of variation, it is desirable to limit data variability, and so in this study we used the transport method to define the disc contour line.

Although previous reports have investigated right-left differences in HRT parameters in healthy human subjects, ${ }^{30} 31$ 

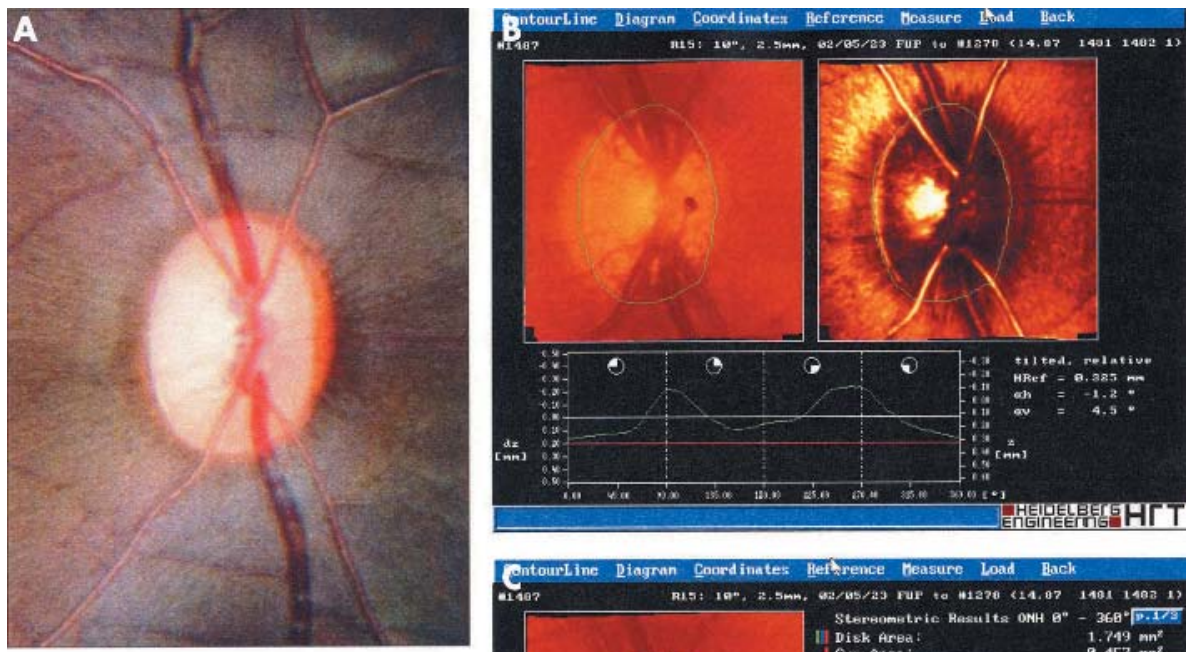

Figure 1 Representative fundus photograph and mean topographic images of optic disc obtained from HRT in normal cynomolgus monkey eye. (A) Fundus photograph; (B) mean topographic images; (C) analysis results for topographic parameters.

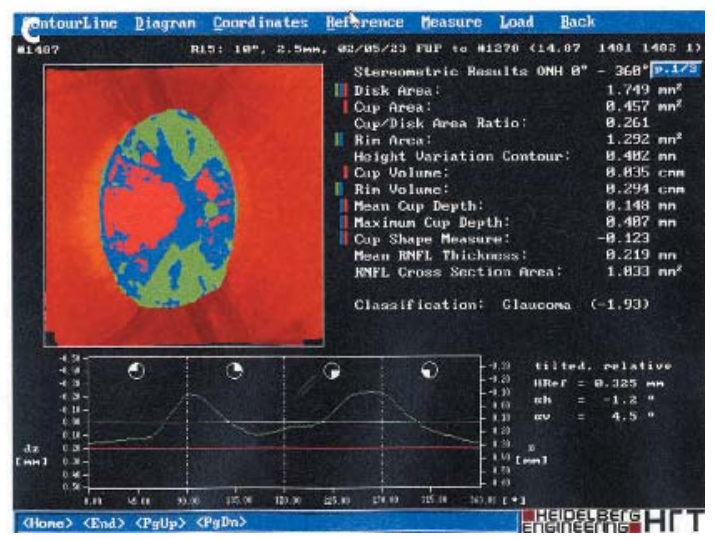

there have been no such reports in normal monkeys (table 5). RV and MnRNFLT were significantly lower in the left eye. ${ }^{31}$ MnRNFLT and RCSA were significantly lower in the right eye. ${ }^{30}$ Although the reason for the discrepancy between these two reports is unclear, the age, sex ratio, and sample size were different between the two studies. By contrast, we observed no significant differences in HRT parameters between right and left eyes in monkeys that were all of one sex (male) and from a narrow age range, although the sample size was much smaller than in the previous reports on humans. ${ }^{30}{ }^{31}$ The values we obtained when we expressed right-left differences as a percentage of the mean value obtained for the right eye (R-L difference ratio) were lower (less than 20\%) for RA, HVC, RV, MnCD, MxCD, CSM, MnRNFLT, and RCSA than for the other parameters.
Therefore, when evaluating longitudinal changes in HRT parameters in experimental glaucoma eyes (using nontreated fellow eyes as controls), these parameters, especially RA and HVC (each of which had an R-L difference ratio of about $10 \%$ ), may be suitable parameters. By contrast, for CA, $\mathrm{C} / \mathrm{D}$, and CV, R-L difference ratios were greater than $40 \%$, suggesting that these parameters are not suitable for this purpose.

Here, correlations among HRT parameters were investigated using Pearson's correlation coefficient, as described elsewhere. ${ }^{32} 33 \mathrm{CA}, \mathrm{C} / \mathrm{D}, \mathrm{CV}$, and $\mathrm{MnCD}$ showed stronger interrelations than the other parameters, a result compatible with previous findings in normal humans. ${ }^{32}$ This supports the optic disc structure of the monkey being comparable to that of humans. HRT parameters are determined following the

Table 2 Optic disc topographic parameters in normal cynomolgus monkey eyes

\begin{tabular}{|c|c|c|c|c|c|c|c|}
\hline \multirow[b]{2}{*}{ Parameters } & \multicolumn{2}{|c|}{ Right eye } & \multicolumn{2}{|l|}{ Left eye } & \multicolumn{3}{|c|}{ Right-left difference } \\
\hline & Mean & SD & Mean & SD & Mean & SD & $\begin{array}{l}\text { As } \% \text { of mean value for right } \\
\text { eye }\end{array}$ \\
\hline Disc area $\left(\mathrm{mm}^{2}\right)$ & 1.62 & 0.24 & 1.62 & 0.24 & 0.08 & 0.06 & 4.9 \\
\hline Cup area $\left(\mathrm{mm}^{2}\right)$ & 0.30 & 0.29 & 0.31 & 0.24 & 0.14 & 0.18 & 46.7 \\
\hline Cup/disc area ratio & 0.17 & 0.15 & 0.18 & 0.12 & 0.08 & 0.09 & 47.1 \\
\hline Rim area $\left(\mathrm{mm}^{2}\right)$ & 1.32 & 0.25 & 1.31 & 0.13 & 0.15 & 0.19 & 11.4 \\
\hline Height variation contour (mm) & 0.40 & 0.05 & 0.39 & 0.05 & 0.04 & 0.03 & 10.0 \\
\hline Cup volume $\left(\mathrm{mm}^{3}\right)$ & 0.03 & 0.05 & 0.03 & 0.03 & 0.02 & 0.03 & 66.7 \\
\hline Rim volume $\left(\mathrm{mm}^{3}\right)$ & 0.33 & 0.10 & 0.31 & 0.06 & 0.06 & 0.07 & 18.2 \\
\hline Mean cup depth (mm) & 0.13 & 0.05 & 0.12 & 0.04 & 0.02 & 0.02 & 15.4 \\
\hline Maximum cup depth (mm) & 0.35 & 0.12 & 0.34 & 0.11 & 0.05 & 0.02 & 14.3 \\
\hline Cup shape measure & -0.17 & 0.06 & -0.16 & 0.06 & 0.03 & 0.04 & -17.6 \\
\hline Mean RNFL thickness (mm) & 0.23 & 0.04 & 0.22 & 0.04 & 0.04 & 0.04 & 17.4 \\
\hline RNFL cross section area $\left(\mathrm{mm}^{2}\right)$ & 1.04 & 0.21 & 0.97 & 0.17 & 0.17 & 0.19 & 16.3 \\
\hline
\end{tabular}

No significant differences between right and left eyes.

Each value represents mean (SD) for 18 eyes. RNFL, retinal nerve fibre layer.

Right-left difference was calculated using the absolute difference between right and left eyes for each animal. 
Table 3 Reproducibility of the optic topographic parameters measured using HRT

\begin{tabular}{lcrrr}
\hline & \multicolumn{3}{l}{ Coefficient of variation } \\
\cline { 2 - 5 } Parameters & Right & SD & Left & SD \\
\hline Cup area $\left(\mathrm{mm}^{2}\right.$ ) & 23.3 & 24.8 & 24.2 & 28.2 \\
Cup/disc area ratio & 23.4 & 24.9 & 24.1 & 28.0 \\
Rim area $\left(\mathrm{mm}^{2}\right.$ ) & 3.4 & 3.4 & 3.1 & 2.3 \\
Height variation contour (mm) & 7.0 & 4.1 & 7.1 & 3.8 \\
Cup volume $\left(\mathrm{mm}^{3}\right.$ ) & 30.7 & 28.7 & 40.9 & 47.3 \\
Rim volume $\left(\mathrm{mm}^{3}\right)$ & 8.3 & 4.7 & 8.0 & 4.8 \\
Mean cup depth (mm) & 7.0 & 4.1 & 8.1 & 7.1 \\
Maximum cup depth (mm) & 6.0 & 4.0 & 5.8 & 5.8 \\
Cup shape measure & 12.6 & 6.3 & 13.1 & 11.1 \\
Mean RNFL thickness (mm) & 7.1 & 5.3 & 7.2 & 4.2 \\
RNFL cross section area (mm ${ }^{2}$ ) & 7.1 & 5.3 & 7.1 & 4.2 \\
\hline
\end{tabular}

Coefficient of variation was obtained from measurement at three times over a 1 week period.

Disc area was defined in the first measurement, and transported to the second and third topographic images. No significant differences between right and left eyes.

Each value represents mean (SD) for 18 eyes. RNFL, retinal nerve fibre layer

Table 4 Correlations among HRT parameters

\begin{tabular}{|c|c|c|c|c|c|c|c|c|c|c|c|c|}
\hline & & CA & $C / D$ & RA & HVC & CV & RV & $M n C D$ & $M \times C D$ & CSM & MnRNFLT & RCSA \\
\hline \multirow[t]{2}{*}{ DA } & right & $0.57^{*}$ & $0.50^{*}$ & 0.31 & 0.32 & $0.48^{*}$ & 0.01 & $0.60^{* *}$ & 0.43 & $0.64^{* *}$ & -0.10 & 0.26 \\
\hline & left & $0.86^{* *}$ & $0.81^{\text {** }}$ & 0.24 & 0.16 & $0.78^{* *}$ & -0.23 & $0.56^{*}$ & 0.42 & 0.42 & -0.30 & 0.08 \\
\hline \multirow[t]{2}{*}{$\mathrm{CA}$} & right & & $0.99^{* *}$ & $-0.61^{\star *}$ & -0.13 & $0.94^{* *}$ & $-0.70^{\star *}$ & $0.87^{* *}$ & $0.74^{* *}$ & $0.65^{\text {** }}$ & $-0.73^{\star *}$ & $-0.53^{*}$ \\
\hline & left & & $0.99^{* *}$ & -0.28 & 0.16 & $0.90^{* *}$ & $-0.59 * *$ & $0.72^{\text {** }}$ & $0.53^{*}$ & $0.64^{* *}$ & $-0.48^{*}$ & -0.17 \\
\hline \multirow[t]{2}{*}{$C / D$} & right & & & $-0.66^{* *}$ & -0.19 & $0.92^{\text {** }}$ & $-0.74^{\star *}$ & $0.89^{* *}$ & $0.77^{* *}$ & $0.64^{* *}$ & $-0.74^{\star *}$ & $-0.56^{*}$ \\
\hline & left & & & -0.36 & 0.17 & $0.87^{* *}$ & $-0.61^{* *}$ & $0.77^{* *}$ & $0.58^{*}$ & $0.67^{* *}$ & -0.47 & -0.17 \\
\hline \multirow[t]{2}{*}{ RA } & right & & & & 0.46 & $-0.62^{* *}$ & $0.81^{* *}$ & -0.43 & -0.43 & -0.13 & $0.74^{\star *}$ & $0.86^{* *}$ \\
\hline & left & & & & $<-0.01$ & -0.24 & $0.69^{* *}$ & -0.32 & -0.21 & -0.43 & 0.36 & $0.48^{*}$ \\
\hline \multirow[t]{2}{*}{ HVC } & right & & & & & -0.07 & $0.54^{*}$ & 0.01 & -0.02 & 0.15 & $0.52^{*}$ & $0.59^{* *}$ \\
\hline & left & & & & & 0.02 & 0.22 & 0.24 & 0.15 & 0.24 & 0.36 & 0.42 \\
\hline \multirow[t]{2}{*}{$C V$} & right & & & & & & $-0.63^{\star *}$ & $0.79^{\text {** }}$ & $0.72^{* *}$ & $0.48^{*}$ & $-0.74^{\star *}$ & $-0.57^{*}$ \\
\hline & left & & & & & & $-0.47^{*}$ & 0.79 ** & $0.71^{\text {** }}$ & 0.32 & -0.35 & -0.07 \\
\hline \multirow[t]{2}{*}{ RV } & right & & & & & & & $-0.55^{\star}$ & $-0.47^{*}$ & -0.44 & $0.89^{* *}$ & $0.87^{* *}$ \\
\hline & left & & & & & & & -0.25 & -0.10 & $-0.59^{* *}$ & $0.77^{* *}$ & $0.72^{* *}$ \\
\hline \multirow[t]{2}{*}{$\mathrm{MnCD}$} & right & & & & & & & & $0.94^{* *}$ & $0.56^{*}$ & $-0.47^{*}$ & -0.26 \\
\hline & left & & & & & & & & $0.94^{\star *}$ & 0.29 & 0.06 & 0.27 \\
\hline \multirow[t]{2}{*}{$M \times C D$} & right & & & & & & & & & 0.27 & -0.37 & -0.23 \\
\hline & left & & & & & & & & & -0.04 & 0.18 & 0.35 \\
\hline \multirow[t]{2}{*}{ CSM } & right & & & & & & & & & & -0.45 & -0.19 \\
\hline & left & & & & & & & & & & -0.45 & -0.30 \\
\hline \multirow[t]{2}{*}{ MnRNFLT } & right & & & & & & & & & & & $0.93^{* *}$ \\
\hline & left & & & & & & & & & & & $0.93^{* *}$ \\
\hline
\end{tabular}

Values are shown as the Pearson correlation coefficient for 18 animals. ${ }^{*} \mathrm{p}<0.05,{ }^{* *} \mathrm{p}<0.01$ (Pearson's correlation test).

$\mathrm{DA}$, disc area; CA, cup area; C/D, cup/disc area ratio; RA, rim area; HVC, height variation contour; CV, cup volume; RV, rim volume; MnCD, mean cup depth;

$M \times C D$, maximum cup depth; CSM, cup shape measure; MnRNFLT, mean RNFL thickness; RCSA, RNFL cross section area; RNFL, retinal nerve fibre layer.

Table 5 HRT parameters in normal human eyes of human in published studies

\begin{tabular}{|c|c|c|c|c|}
\hline & \multicolumn{2}{|c|}{ Herman et $a l^{31}$} & \multicolumn{2}{|l|}{ Ghergel et $a l^{30}$} \\
\hline & \multicolumn{2}{|c|}{154 (female), 728 (male) } & \multicolumn{2}{|c|}{80 (female), 77 (male) } \\
\hline & \multicolumn{2}{|c|}{ Age range $35-70$ years } & \multicolumn{2}{|c|}{ Age range $14-77$ years } \\
\hline & Right & Left & Right & Left \\
\hline $\begin{array}{l}\text { Disc area }\left(\mathrm{mm}^{2}\right) \\
\text { Cup area }\left(\mathrm{mm}^{2}\right) \\
\text { Cup/disc area ratio } \\
\text { Rim area }\left(\mathrm{mm}^{2}\right) \\
\text { Cup volume }\left(\mathrm{mm}^{3}\right) \\
\text { Rim volume }\left(\mathrm{mm}^{3}\right) \\
\text { Maximum cup depth }(\mathrm{mm}) \\
\text { Cup shape measure } \\
\text { Mean RNFL thickness }(\mathrm{mm}) \\
\text { RNFL cross section area }\left(\mathrm{mm}^{2}\right)\end{array}$ & $\begin{array}{l}1.83(0.39) \\
0.44(0.32) \\
0.22(0.13) \\
1.39(0.27) \\
\text { NA } \\
0.38(0.13) \\
\text { NA } \\
\text { NA } \\
0.26(0.07) \\
\text { NA }\end{array}$ & $\begin{array}{l}1.81(0.39) \\
0.44(0.32) \\
0.22(0.13) \\
1.37(0.27) \\
\text { NA } \\
0.36(0.12) \\
\text { NA } \\
\text { NA } \\
0.25(0.07) \\
\text { NA }\end{array}$ & $\begin{array}{r}1.92(0.38) \\
0.49(0.26) \\
0.24(0.10) \\
1.43(0.28) \\
0.12(0.10) \\
0.37(0.12) \\
0.66(0.18) \\
-0.23(0.07) \\
0.25(0.06) \\
1.20(0.32)\end{array}$ & $\begin{array}{l}1.90(0.37) \\
0.47(0.28) \\
0.23(0.11) \\
1.42(0.25) \\
0.11(0.11) \\
0.40(0.13) \\
0.69(0.18) \\
-0.24(0.07) \\
0.27(0.06) \\
1.33(0.32)\end{array}$ \\
\hline
\end{tabular}

Each value represents mean (SD). RNFL, retinal nerve fibre layer.

NA, not available.

Data in table are derived from previous reports by Herman et $a^{31}$ and Ghergel et al ${ }^{30}$ (see column headings). 
drawing of contour lines to define DA. This procedure is to some extent observer dependent. Therefore, it is important to know which parameters are influenced by DA. In our study, DA correlated with CA, C/D, CV and MnCD (in both eyes), and CSM (in the right eye only). Further, for most of these parameters both the coefficient of variation and R-L difference ratio were relatively high. By contrast, RA, HVC, RV, MxCD, MnRNFLT, and RCSA seemed to be unaffected by DA in either eye. For these parameters, both the coefficient of variation (less than $10 \%$ ) and the R-L difference ratio (less than $20 \%$ ) were relatively low. These findings suggest that these parameters, especially RA and HVC may be more suitable than the other parameters for evaluating optic disc changes.

When evaluating HRT parameters in another non-human primate (for example, rhesus monkey) under our experimental conditions, our results (in particular, coefficient of variation) may be useful. However, as previous reports have indicated that the optic structures measured by HRT display racial differences, ${ }^{34}{ }^{35}$ it is possible that the monkey optic disc structure also differs among the various kinds of monkeys.

In conclusion, we examined optic disc topographic parameters in normal eyes in male cynomolgus monkeys of similar ages. RA and HVC showed relatively low coefficient of variation values (less than $10 \%$ ), low R-L difference ratios (about 10\%), no correlation with DA in either eye, and relatively weak interrelations. These basic results may be useful when HRT is used in monkeys to evaluate optic disc changes in experimentally induced optic disc damage and also to evaluate the efficacy of potential treatments.

\section{Authors' affiliations}

T Taniguchi, M Sasaoka, Research and Development Center, Santen Pharmaceutical Co Ltd, Nara, Japan

M Shimazawa, H Hara, Department of Biofunctional Molecules, Gifu Pharmaceutical University, Gifu, Japan

M Araie, G Tomita, Department of Ophthalmology, University of Tokyo School of Medicine, Tokyo, Japan

Y Kitazawa, Department of Ophthalmology, Gifu University School of Medicine, Gifu, Japan

\section{REFERENCES}

1 Gaasterland D, Kupfer C. Experimental glaucoma in the rhesus monkey. Invest Ophthalmol Vis Sci 1974;13:455-7.

2 Varma R, Quigley HA, Pease ME. Changes in optic disk characteristics and number of nerve fibers in experimental glaucoma. Am J Ophthalmol 1992; 114:554-9.

3 Hayreh SS, Pe'er J, Zimmerman MB. Morphologic changes in chronic highpressure experimental glaucoma in rhesus monkeys. J Glaucoma 1999;8:56-71.

4 Jonas JB, Hayreh SS. Localised retinal nerve fibre layer defects in chronic experimental high pressure glaucoma in rhesus monkeys. Br J Ophthalmol 1999:83:1291-5.

5 Quigley HA, Hohman RM, Addicks EM, et al. Blood vessels of the glaucomatous optic disc in experimental primate and human eyes. Invest Ophthalmol Vis Sci 1984;25:918-31.

6 Derick RJ, Pasquale LR, Pease ME, et al. A clinical study of peripapillary crescents of the optic disc in chronic experimental glaucoma in monkey eyes. Arch Ophthalmol 1994;112:846-50.

7 Hayreh SS, Jonas JB, Zimmerman MB. Parapapillary chorioretinal atrophy in chronic high-pressure experimental glaucoma in rhesus monkeys. Invest Ophthalmol Vis Sci 1998;39:2296-303.

8 Jonas JB, Hayreh SS. Optic disk morphology in experimental central retinal artery occlusion in rhesus monkeys. Am J Ophthalmol 1999;127:523-30.
9 Jonas JB, Hayreh SS. Influence of experimental chronic high-pressure glaucoma on age-related macular degeneration in rhesus monkeys. Invest Ophthalmol Vis Sci 2000;41:2972-7.

10 Jonas JB, Hayreh SS. Ophthalmoscopic appearance of the normal optic nerve head in rhesus monkeys. Invest Ophthalmol Vis Sci 2000;41:2978-83.

11 Varma R, Steinmann WC, Scott IU. Expert agreement in evaluating the optic disc for glaucoma. Ophthalmology 1992;99:215-21.

12 Malinovsky VE. An overview of the Heidelberg retina tomograph. J Am Optom Assoc 1996:67:457-67.

13 Ahn BS, Kee C. Ability of a confocal scanning laser ophthalmoscope (TopSS) to detect early glaucomatous visual field defect. $\mathrm{Br} J$ Ophthalmol 2000;84:852-5.

14 Azuara-Blanco A, Harris A, Cantor LB. Reproducibility of optic disk topographic measurements with the Topcon ImageNet and the Heidelberg retina tomograph. Ophthalmologica 1998;212:95-8

15 lester M, Mikelberg FS, Courtright $P$, et al. Correlation between the visual field indices and Heidelberg retina tomograph parameters. J Glaucoma 1997;6:78-82.

16 Eid TM, Spaeth GL, Katz $\sqcup$, et al. Quantitative estimation of retinal nerve fiber layer height in glaucoma and the relationship with optic nerve head topography and visual field. J Glaucoma 1997;6:221-30.

17 Lan YW, Henson DB, Kwartz AJ. The correlation between optic nerve head topographic measurements, peripapillary nerve fibre layer thickness, and visual field indices in glaucoma. Br J Ophthalmol 2003;87:1 135-41.

18 Heickell AG, Bellezza AJ, Thompson HW, et al. Optic disc surface compliance testing using confocal scanning laser tomography in the normal monkey eye. J Glaucoma 2001;10:369-82.

19 Burgoyne CF, Mercante DE, Thompson HW. Change detection in regional and volumetric disc parameters using longitudinal confocal scanning laser tomography. Ophthalmology 2002;109:455-66.

20 Ervin JC, Lemij HG, Mills RP, et al. Clinician change detection viewing longitudinal stereophotographs compared to confocal scanning laser tomography in the LSU Experimental Glaucoma (LEG) Study. Ophthalmology 2002; 109:467-81.

21 Yücel YH, Gupta N, Kalichman MW, et al. Relationship of optic disc topography to optic nerve fiber number in glaucoma. Arch Ophthalmol 1998;116:493-7.

22 Hare WA, WoldeMussie E, Weinreb RN, et al. Efficacy and safety of memantine treatment for reduction of changes associated with experimental glaucoma in monkey, II: Structural measures. Invest Ophthalmol Vis Sci 2004;45:2640-51

23 Quigley HA, Pease ME. Change in the optic disc and nerve fiber layer estimated with the glaucoma-scope in monkey eyes. J Glaucoma 1996:5:106-16.

24 Shirakashi M, Abe H, Sawaguchi S, Iwata K. The relationship between deterioration and reversal of optic disc cupping in monkeys with chronic experimental high-pressure glaucoma. Graefes Arch Clin Exp Ophthalmol 1998;236:546-52.

25 Burgoyne CF, Varma R, Quigley HA, et al. Global and regional detection of induced optic disc change by digitized image analysis. Arch Ophthalmol 1994; 112:261-8.

26 Burgoyne CF, Quigley HA, Thompson HW, et al. Measurement of optic disc compliance by digitized image analysis in the normal monkey eye. Ophthalmology 1995; 102:1790-9.

27 Saruhan A, Orgul S, Kocak I, et al. Descriptive information of topographic parameters computed at the optic nerve head with the Heidelberg retina tomograph. J Glaucoma 1998;7:420-9.

28 Tan JC, Garway-Heath DF, Hitchings RA. Variability across the optic nerve head in scanning laser tomography. Br J Ophthalmol 2003;87:557-9.

29 Roff EJ, Hosking SL, Barnes DA. The influence of contour line size and location on the reproducibility of topographic measurement with the Heidelberg retina tomograph. Ophthalmic Physiol Opt 2001;21:173-81.

30 Gherghel D, Orgul S, Prunte C, et al. Interocular differences in optic disc topographic parameters in normal subjects. Curr Eye Res 2000;20:276-82.

31 Hermann MM, Theofylaktopoulos I, Bangard N, et al. Optic nerve head morphometry in healthy adults using confocal laser scanning tomography. Br J Ophthalmol 2004;88:761-5.

32 Nakamura H, Maeda T, Suzuki Y, et al. Scanning laser tomography to evaluate optic discs of normal eyes. Jpn J Ophthalmol 1999;43:410-14.

33 Durukan AH, Yucel I, Akar Y, et al. Assessment of optic nerve head topographic parameters with a confocal scanning laser ophthalmoscope. Clin Experiment Ophthalmol 2004;32:259-64.

34 Tsai CS, Zangwill L, Gonzalez C, et al. Ethnic differences in optic nerve head topography. J Glaucoma 1995;4:248-57.

35 Girkin CA, McGwin G Jr, McNeal SF, et al. Racial differences in the association between optic disc topography and early glaucoma. Invest Ophthalmol Vis Sci 2003;44:3382-7. 